\title{
Editorial
}

Nephrology

\section{Cortical or Trabecular Bone: What's the Difference?}

\author{
Susan M. Ott \\ Department of Medicine, University of Washington, Seattle, WA, USA
}

Bone is an exquisite example of the principle that form follows function. Bone provides structural support for ambulation and ventilation; a reservoir for calcium, phosphate, amino acids, and bicarbonate; protection of interior organs; transmission of sound waves; and nurturing of hematopoietic stem cells. Metabolic functions include secretion of hormones that regulate both mineral and energy metabolism. To accomplish these functions, the bone has cortical and trabecular compartments.

Approximately $80 \%$ of the bone mass is in the cortical compartment. Vascular channels occupy about $30 \%$ of the volume. The surface to volume ratio in cortical bone is much lower than in trabecular bone. With aging or disease, the cortex becomes more porous, thus gaining surface area but losing strength. In the long bones, increased porosity near the periosteal surface causes more loss of strength than increased porosity near the endocortical surface. Slow periosteal expansion throughout life partially compensates for this loss of strength, because the bending strength is proportional to the radius to the fourth power.

In the trabecular compartment, $20 \%$ of the volume is composed of bone, and the remaining space is filled with

\section{KARGER}

() 2018 S. Karger AG, Basel

E-Mail karger@karger.com

www.karger.com/ajn marrow and fat. Trabecular bone transfers mechanical loads from the articular surface to the cortical bone. The hydraulic properties absorb shock. The material properties of the bone compartments differ: trabecular bone has lower calcium content and more water content compared to cortical bone. Trabecular bone has a large surface exposed to the bone marrow and blood flow, and the turnover is higher than in cortical bone [1]. Resorption takes place along bone surfaces in the trabecular bone, whereas in the cortical bone, resorption tunnels through the bone itself. The endocortical surface which is exposed to the marrow as well as the solid bone often has higher bone turnover than other trabecular or cortical surfaces.

Some have felt that there was too much focus on trabecular bone loss whereas others have emphasized the contributions of trabecular bone. In fact, both cortical and trabecular bone are important to bone strength, and the relationships are complex. The vertebral body is the classical trabecular bone site, and vertebral compression fractures are the hallmark of osteoporosis. However, the thin cortical shell plays a substantial role.

The hip is considered a cortical bone site, but both cortical and trabecular bone contribute to femoral strength, 
the contribution of the cortical bone being higher in femurs with lower trabecular bone density [2]. Furthermore, the cortical bone supports bending in the distal region of the femoral neck and the trabecular bone supports the proximal load.

Signals to the bone, either mechanical or chemical, can differentially affect the bone compartments. The effects are complicated by gender, age, or disease. For example, bed rest studies in healthy men show that during disuse, bone is lost more from the trabecular compartment than from the cortex at the tibia [3].

Low intensity vibration in patients with chronic kidney disease increases strength parameters of the tibia due to changes in the trabecular compartment but not the cortex [4].

The gonadal hormones differentially affect the bone compartments, depending on gender. In both men and women, testosterone supports periosteal bone expansion while estrogen prevents cortical bone loss. Trabecular bone loss is prevented by estrogen in women and testosterone in men.

Bone loss after menopause is more rapid in the trabecular bone, but because the cortical bone comprises $80 \%$ of the skeleton, the absolute amount of bone loss is similar from each compartment for the first 10 years. Later, there is more loss from cortical bone. The Haversian canals widen, increasing the surface/volume ratio of the bone and further enhancing loss from bone that was formerly cortical but has become trabecularized [5]. In women, large canals causing cortical porosity are present throughout the cortex, whereas they are closer to endocortex in men. Overall, women lose more cortical bone then men, but trabecular bone loss is similar. With aging, the diameter of long bones increases more in men than women, which increases the bending strength.

Trabecular and cortical bone can be differentially affected by hormones and medications. Daily injections of parathyroid hormone (PTH) decrease the cortical bone mass and increase in trabecular bone mass. The significant increase in strength is attributable to the increase in trabecular bone.

In primary hyperparathyroidism, bone is lost from the cortical compartment with reduced cortical thickness and increased porosity. The trabecular compartment, particularly in the axial skeleton, has normal bone density. In patients with chronic kidney disease, the higher PTH levels are associated with rapid cortical bone loss at the radius, but the trabecular density and microarchitecture is preserved [6].
A new study published in this issue further emphasizes the importance of cortical bone in renal osteodystrophy. Sharma et al. [7] preformed iliac crest bone biopsies in 14 patients and measured structural parameters using both standard histomorphometry (on 2-dimensional sections) and micro-CT on the core sample (3-dimensional). They found normal trabecular parameters but decreased cortical thickness and increased cortical porosity.

Sharma et al. [7] suggested that the cortical component of bone volume has been relatively ignored in bone histomorphometric studies of renal osteodystrophy. However, at the Kidney Disease: Improving Global Outcomes consensus conference about this topic, cortical bone was included under the aspect of the bone volume, and several important biopsy studies have included cortical thickness as one of the reported measurements. The lack of focus on this cortex could be due to the limited sample size of the biopsy and questionable correlations to the overall cortical bone mass in the skeleton.

Bone biopsies are performed on the iliac crest because it is a safe and convenient anatomic location. However, it may not be representative of the skeleton. The iliac crest is not weight bearing, and the trabecular bone has higher bone turnover than in the metaphysis of the long bones. The cortex is thinner than in long bones and there is not as much load bearing as in the axial skeleton.

High resolution peripheral quantitative CT (HR-pQCT) is a non-invasive technique that can separate cortical from trabecular components in the radius or tibia, and can measure some aspects of the trabecular microarchitecture. A study of 54 women measured the cortical thickness and porosity on bone biopsies by histomorphometry and the trabecular parameters on the core biopsy with micro-CT, and performed HR-pQCT on the radius and tibia. The correlations between measurements on the iliac crest biopsies and on the peripheral bones were only modest [8].

HR-pQCT has been used in dialysis patients, demonstrating that cortical thickness and area were the parameters most reduced and were related to PTH. The reduction in the trabecular parameters at the radius was worse in women than in men [9].

Thus, although invasive bone biopsy remains the gold standard for determination of bone mineralization and turnover, it is probably not the best measure of bone volume. Clearly the cortex is an important contributor of bone strength. The use of micro-CT in dialysis patients is novel and this allows more precise definition of the bone structure than the 2-dimensional histology. However, 
both techniques are limited by the small size of the bone sample and the location of the biopsy. Bone biopsies are invasive and both micro-CT and HR-pQCT require expensive equipment not readily available for clinical use. Although not as precise, dual energy radiographic densitometry measurements of the radius provide a measure of cortical bone loss that is practical and widely available, but we lack prospective studies in dialysis patients that have adequate power to determine relationship to skeletal fractures.

\section{Disclosure Statement/Funding Source}

None.

\section{References}

1 Parfitt AM: Misconceptions (2): turnover is always higher in cancellous than in cortical bone. Bone 2002;30:807-809.

-2 Johannesdottir F, Thrall E, Muller J, Keaveny TM, Kopperdahl DL, Bouxsein ML: Comparison of non-invasive assessments of strength of the proximal femur. Bone 2017;105:93-102.

-3 Cervinka T, Sievanen H, Hyttinen J, Rittweger J: Bone loss patterns in cortical, subcortical, and trabecular compartments during simulated microgravity. J Appl Physiol 2014;117: 80-88.

4 Rajapakse CS, Leonard MB, Kobe EA, Slinger MA, Borges KA, Billig E, et al: The efficacy of low-intensity vibration to improve bone health in patients with end-stage renal disease is highly dependent on compliance and muscle response. Acad Radiol 2017;24:1332-1342.

5 Seeman E: Age- and menopause-related bone loss compromise cortical and trabecular microstructure. J Gerontol A Biol Sci Med Sci 2013;68:1218-1225.

6 Nickolas TL, Stein EM, Dworakowski E, Nishiyama KK, Komandah-Kosseh M, Zhang $\mathrm{CA}$, et al: Rapid cortical bone loss in patients with chronic kidney disease. J Bone Miner Res 2013;28:1811-1820.

7 Sharma AK, Toussaint ND, Masterson R, et al: Deterioration of cortical bone microarchitecture: critical component of renal osteodys- trophy evaluation. Am J Nephrol 2018;47: 376-384.

8 Cohen A, Dempster DW, Muller R, Guo XE, Nickolas TL, Liu XS, et al: Assessment of trabecular and cortical architecture and mechanical competence of bone by high-resolution peripheral computed tomography: comparison with transiliac bone biopsy. Osteoporos Int 2010;21:263-273.

9 Negri AL, Del Valle EE, Zanchetta MB, Nobaru M, Silveira F, Puddu M, et al: Evaluation of bone microarchitecture by high-resolution peripheral quantitative computed tomography (HR-pQCT) in hemodialysis patients. Osteoporos Int 2012;23:2543-2550. 